\title{
A Virtual Prototyping Technology for Design of Pressing Equipment of Dried Tofu
}

\author{
Mingji Huang ${ }^{1, *}$ and Xiuping Dong ${ }^{2}$ \\ ${ }^{1}$ Department of Mechanical Engineering, University of Science and Technology Beijing, \\ Beijing, P.R. China 100083 \\ Tel.: +86-010-62332538; Fax: +86-010-62329145 \\ huangmingji@263. net \\ ${ }^{2}$ Department of Mechanical Engineering, Beijing Technology and Business University, \\ Beijing, P.R. China 100048
}

\begin{abstract}
The industry of dried tofu products wants to achieve breakthrough, the key lies in developing efficient processing equipments. Pressing equipment of dried tofu has been mostly designed according to the line -"manual design (some with a CAD) - prototype production - prototype testing - design modification". However this way not only gets a high cost of research, but also a long cycle. It used virtual prototyping technology, at first, according to the design requirements, created three-dimensional modeling by using Pro/E software ; at last, made the feasibility analysis of kinematics and dynamics by using of $\mathrm{AD}$ AMS software. Through optimization design, analysis and control of cost, it had several characters as follows, simple machine, low cost, high productivity, it had great significance to mechanization and industrialization of pressing equipment of dried tofu.
\end{abstract}

Keywords: virtual prototyping, bean product, Pro/E, ADAMS.

\section{Introduction}

Nowadays, the production of soybean products, industrial automation degree of production is still relatively backward (Jiang Lianzhou et al., 2007; Wu Yuefang et al., 2006), especially dried tofu production; most manufacturers remain handicraft workshop. Mechanized processing and industrialized processing are slow and tortuous, expect the market factors, the weakness of technical force and the lack of advanced research and technology is a major obstacle. Although the production of dried tofu has a long history and accumulated rich production experience, some experience has not been theoretical and some phenomenon can not be explained by the theory. Pressing equipment of dried tofu has been mostly designed according to the line -"manual design (some with a CAD) - prototype production - prototype testing - design modification". However this way not only gets a high cost of research, but also a long cycle. Virtual prototyping technology provides a new means for the design of equipment. The paper is application research of virtual prototyping technology in the design of production equipment of dried tofu.

\footnotetext{
* Corresponding author.
} 


\section{System of Virtual Prototyping}

It chooses Pro/E and ADAMS to build the research environment of virtual prototyping. In Mechanical/Pro modules, not only moving parts and sports relations can be defined, but also there is data conversion interface with ADAMS(Basset $\mathrm{M}$ et al., 2002), Although ADAMS has a powerful simulation of kinematics and dynamics, its 3D modeling capabilities and interoperability are weak. The system uses Pro/E to carry out 3D modeling and Mechanical/Pro module to definite constraint. Block diagram of virtual prototyping system is shown in Fig.1 (Zorriassatine F et al., 2003; Xiong Guangleng et al., 2001), the prototype is shown in Fig.2.

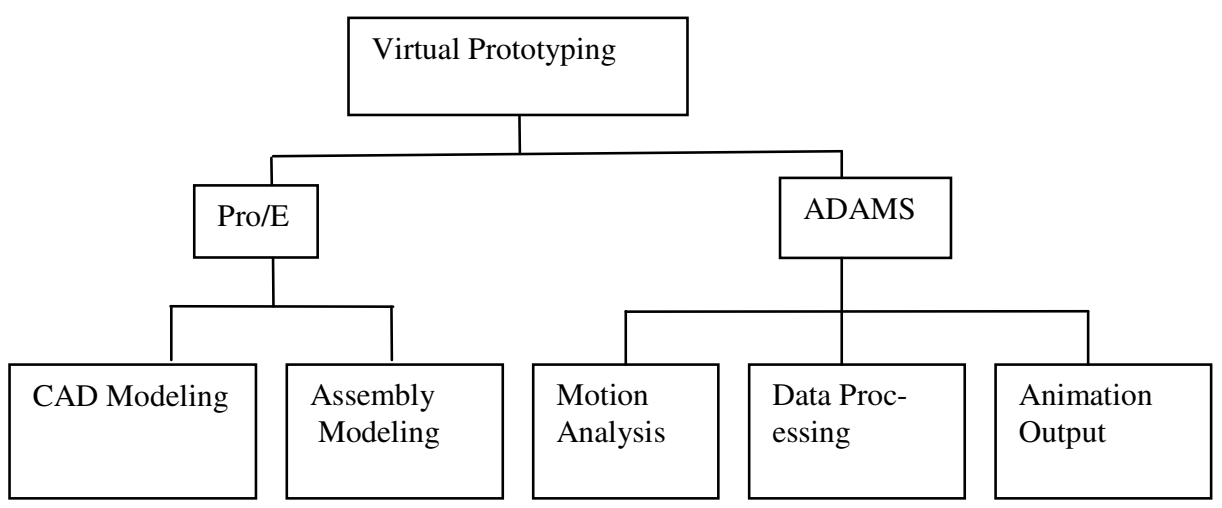

Fig. 1. Virtual prototyping system

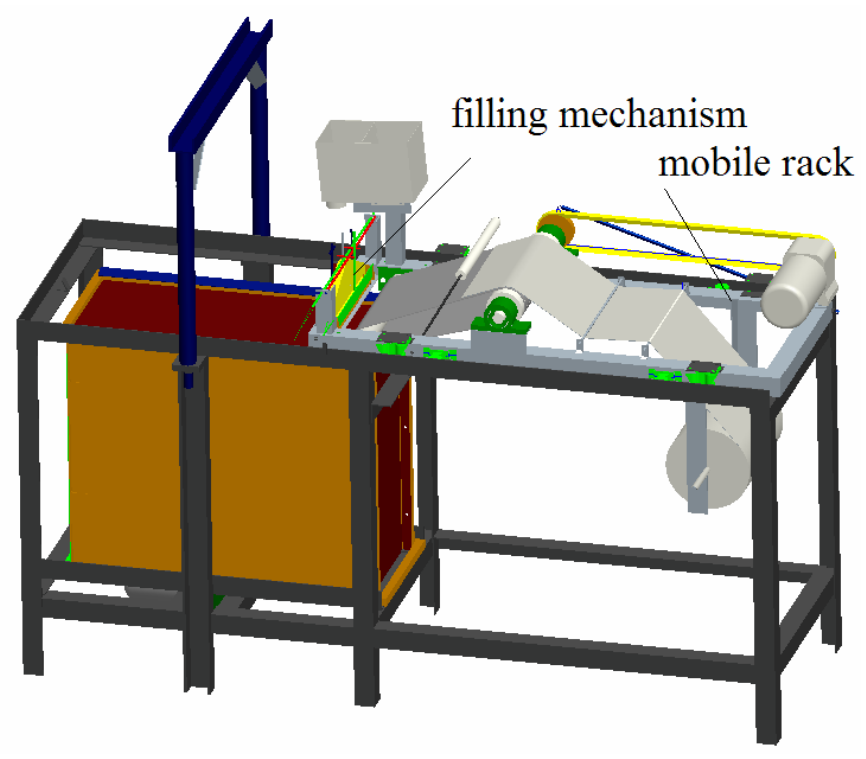

Fig. 2. Prototype of Pressing Equipment 


\section{Kinematics and Dynamics Analysis of Crucial Element}

\subsection{Kinematic Analysis}

Even laying of mobile platforms is one of the key mechanical structure of pressing equipment of dried tofu, the carrying of the machine body, other key mechanical structure are placed on the mobile platform. Mobile platform is moving back and forth driven by the rocker, the rocker connects with the belt through the straight hinge, as shown in Fig.3.

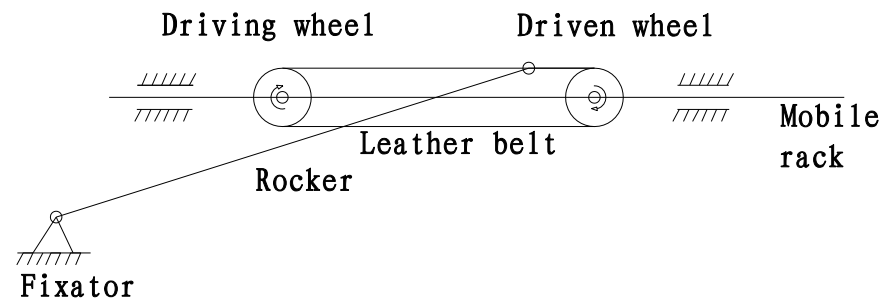

Fig. 3. Schematic drawing of motion principle of mobile rack

The velocity simulation of rocker centroid in the vertical plane is shown in Fig.4 using ADAMS, the interception of their 10 seconds of the motion diagrams, the abscissa is time(s), ordinate is speed $(\mathrm{mm} / \mathrm{s})$. Straight-line describes the level of the ground-rocker is relatively static, subfoveal and protruding are the part of movement, the highest and the lowest point is the point that the mobile platform changes the movement direction.

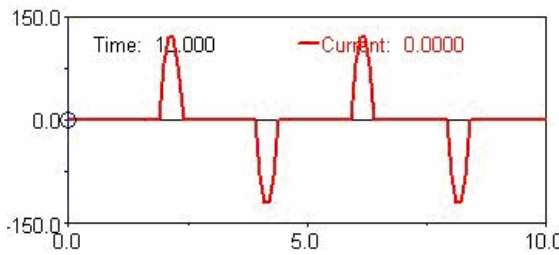

Fig. 4. velocity of the rocker centroid

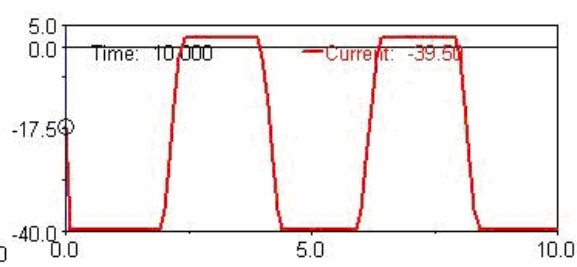

Fig. 5. position of the rocker centroid

Rocker centroid position in the vertical plane is shown in Fig.5, abscissa is time(s), ordinate for the high $(\mathrm{mm})$, because the upper surface of the mobile platform is defined as zero-plane in the assembly drawing, rocker is on the top of the mobile platform, its moving symmetrical line isn't at 0 , Fig. 5 shows the moving range of rocker centroid is $42 \mathrm{~mm}$, we can see the moving range of the rocker and the belt hinge's place is $84 \mathrm{~mm}$, the diameter of the pulley is $80 \mathrm{~mm}$, in addition hinge place $2 \mathrm{~mm}$, moving range of the rocker at the hinge place is just $84 \mathrm{~mm}$.

The upper horizon line in the Fig.5 shows the hinge point of the rocker and the belt is at loose side of belt, the lower horizon shows the hinge point of the rocker and the 


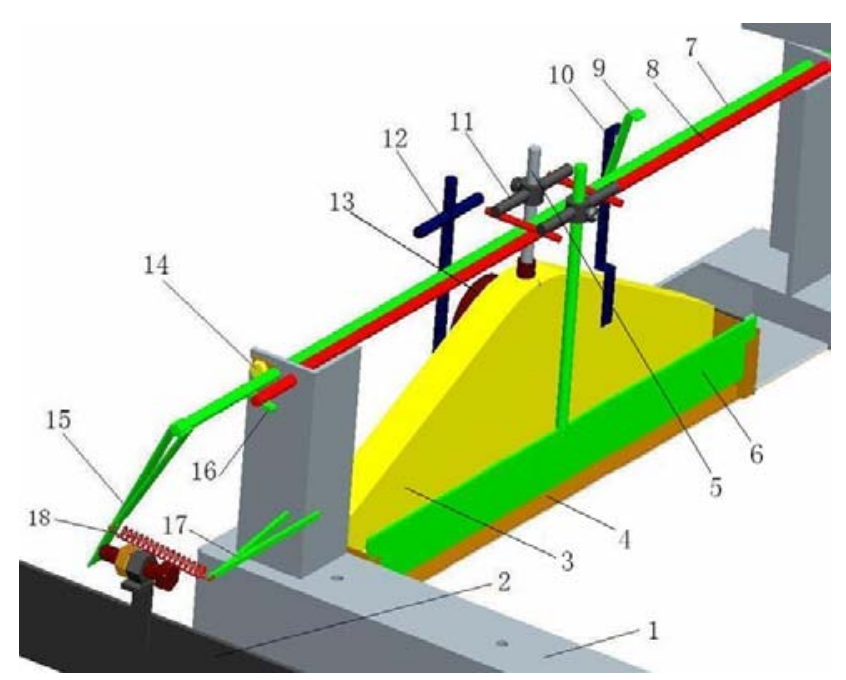

1. movable rack, 2. big frame, 3. upper material bucket, 4.lower material bucket, 5. upper material bucket valve, 6. lower material bucket valve, 7. rotary rod, 8.holding rod, 9.rotation hook, 10. material bucket hook,11.valve location rod, 12. portable rod, 13.feeding hole, 14.jack bolt, 15.impact tablets, 16.stop rotation tablets, 17.spring fixed flap, 18. tension spring。

Fig. 6. The overall drawing of filling mechanism

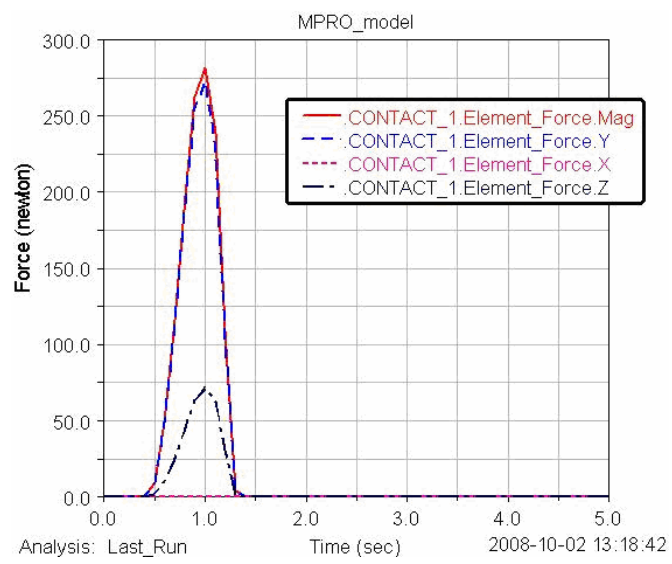

Fig. 7. Whereabouts high of drop-off material bucket and the opened valve

belt is at tight side of belt, the phase of the curve between upper and lower shows the centroid moving of the hinge point of rocker and belt on the pulley.

In the process of valve opening, the centroid trajectory of the drop-off material bucket and material bucket valve is shown in Fig.7, abscissa is time(s), ordinate is the height $(\mathrm{mm})$, solid line is the centroid motion trajectories of the drop-off material bucket, the dashed line is the centroid motion trajectories of drop-off material bucket valve. the height of the valve to open is approximately $9 \mathrm{~mm}$ from the figure. 


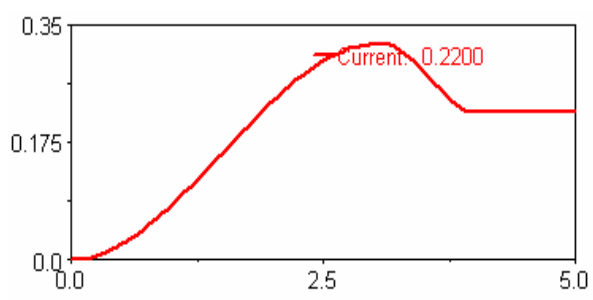

Fig. 8. rotation angle survey chart of rotary rod

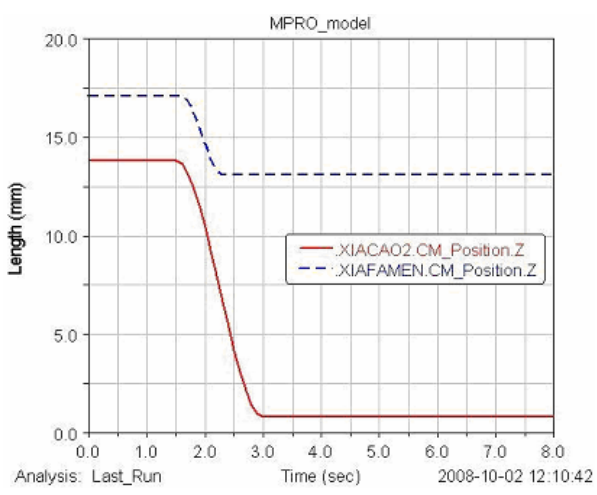

Fig. 9. Impact force of impact tablet and rotary rod

Fig. 8 is the rotation angle survey chart of rotary rod, abscissa is time (s), ordinate is the rotation angle ( $\mathrm{rad})$, rotating rod turns soon when it rotates to a certain point, at this time the rotation is drop-off material bucket has fallen off, the entire movable device moves in the opposite direction.

\subsection{Kinetic Analysis}

The simulation can measure the collision force of the rotary rod and the big frame, and Fig.9 shows the collision force of the collision tablets on the rotary rod by, solid line shows resultant force, long-dashed line shows $\mathrm{Y}$ force, and short dashed line shows $\mathrm{X}$ force, and the center line shows $\mathrm{Z}$ force. the greatest collisions force of is about $260 \mathrm{~N}$.

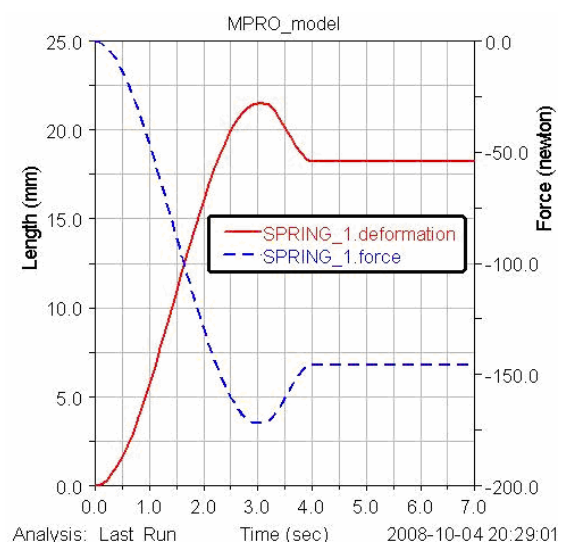

Fig. 10. Spring length and tension

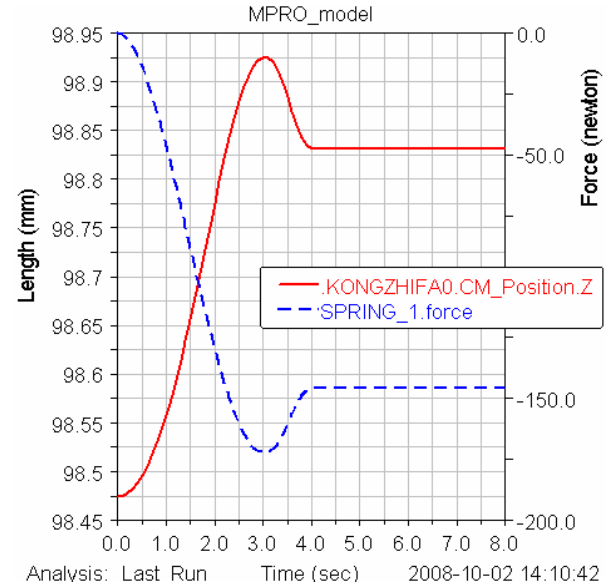

Fig. 11. Position of rotary rod and spring tension 
Fig.10 shows spring length and tension changes, the solid line shows the spring length, corresponding to the left ordinate $(\mathrm{mm})$, dashed curve shows the spring tension, corresponding to the right ordinate $(\mathrm{N})$. Spring stiffness coefficient is $800 \mathrm{~N} / \mathrm{m}$, in accordance with changes in the length of the spring can be calculated the largest spring rally is about $170 \mathrm{~N}$, and the spring tension curve also showed the biggest rally is about $170 \mathrm{~N}$.

Fig.11 shows position of rotary rod and spring tension, the solid line shows the position of rotary rod, corresponding to the left ordinate $(\mathrm{mm})$, dashed curve shows spring tension, corresponding to the right ordinate $(\mathrm{N})$. the figure of the spring force and the figure of the position of rotary rod is similar ,and the figure of the position of rotary rod and the figure of the rotation angle curve chart of rotary rod is similar (see Fig. 7).

\section{Simulation and Discussion}

When we assemble in the Pro/E, certain order must be complied. The first installation of spare parts must be carefully chosen, because the other parts are based on the first, and the default location of the first parts can not be changed, if changing, the remaining parts will change accordingly. Because some parts should be inserted into other

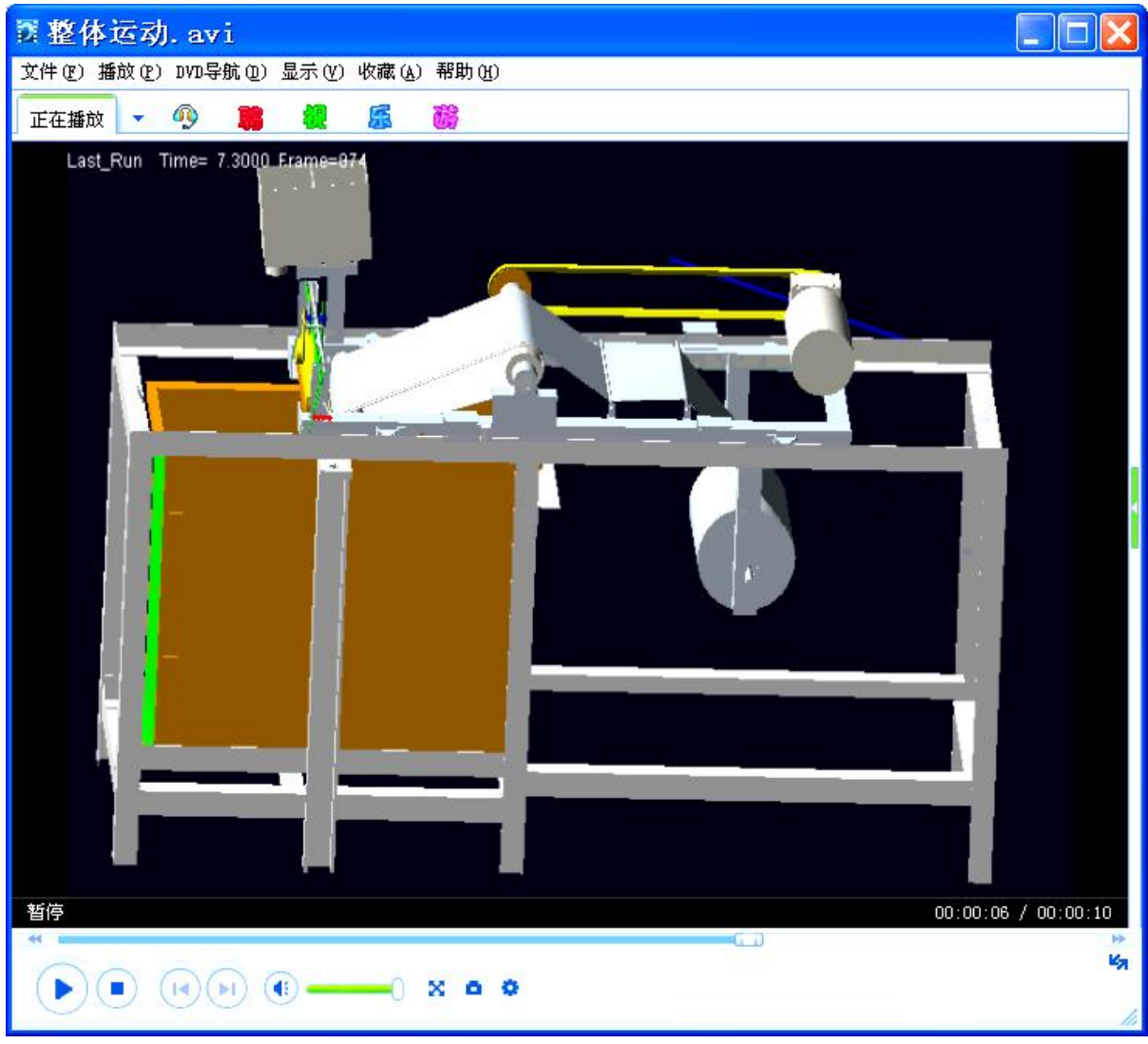

Fig. 12. Movement of dried tofu machine state diagram 
parts of the location, we must pay attention to the part which is based on (the parent parts), the parts which is inserted are the sub-items, which should be moved with the parent parts, of course, there may be no parent parts when parts are assembled directly to the coordinates of drawing, so that even if changes happen in other parts, it will not be affected. The final assemble drawing in Pro/E is shown in Fig.2. Then the drawing is conversed to ADAMS by connecting software Mechanical/Pro. The various constraints and the power source can be directly loaded in Pro/E, and converted to the ADAMS or loaded directly in ADAMSADAMS/View.

In ADAMS/View, every part loaded has to be definite, if it does not affect the visual effects, animation and analysis, we should make the amount of parts to be the fewest in ADAMS/View. If there is no relative motion and no mechanical interference of two parts, we can connect the two parts into a part, so that the model can be simplified without affecting the results of simulation analysis.

It can make movement simulation and analysis after finishing a variety of constraints and drivers, ADAMS can output the results of the animation and analysis by post-processing module of ADAMS/PostProcessor. By comparing the test data and simulation results, ADAMS simulation can also verify the validity of the analysis, carry on mathematics operation to the data result and statistical analysis of the output. Fig.12 is the dummy specimen simulation animation which records from ADAMS/PostProcessor, The next work is to gathering test data, comparing to the simulation result.

\section{Conclusion}

It applies virtual prototyping technology in design of dried tofu production equipment, chooses Pro/E and ADAMS to build virtual prototyping, has overcome the insufficiency of ADAMS in 3D modeling, meanwhile it is highly effective to establishing virtual prototyping and accurate using Mechanical/Pro and the ADAMS data conversion, it has realized the entire virtual movement through the ADAMS simulation. The findings have the vital significance for design of pressing equipment of dried tofu, also has the reference value to similar products' development.

\section{References}

Basset, M., Pearson, R., Fleming, N., et al.: An approach to model-based design. In: The 2002 North American ADAMS Users Conference in Scottsdale, Arizona (2002)

Lianzhou, J., Shaoxin, H.: Status and Suggestions for the Development of Soybean Processing Industry in China. Journal of Agricultural Science and Technology 9(06), $22-27$ (2007) (in Chinese)

Yuefang, W.: Development of Chinese Bean Food Trade. For the Food \& Beverage Industry (04), 38-39 (2006) (in Chinese)

Zorriassatine, F., Wykes, C., Parkin, R., et al.: A survey of virtual prototyping techniques for mechanical product development. Proceedings of I Mech. E. Part B:J. Engineering Manufacture 217(1), 513-530 (2003)

Guangleng, X., Bohu, L., Xudong, C.: Virtual Prototyping Technology. Journal of System Simulation 13(1), 114-117 (2001) (in Chinese) 